\title{
LSKL peptide alleviates subarachnoid fibrosis and hydrocephalus by inhibiting TSP1-mediated TGF- $\beta 1$ signaling activity following subarachnoid hemorrhage in rats
}

\author{
FAN LIAO $^{1}$, GAOFENG LI $^{2}$, WEN YUAN ${ }^{3}$, YUJIE CHEN $^{4}$, YUCHUN ZUO $^{1}$, \\ KAUTHAR RASHID ${ }^{1}$, JOHN H. ZHANG ${ }^{5}$, HUA FENG $^{4}$ and FEI LIU ${ }^{1}$ \\ ${ }^{1}$ Department of Neurosurgery, The Third Xiangya Hospital, Central South University, Changsha, Hunan 410013; \\ Departments of ${ }^{2}$ Oncology and ${ }^{3}$ Neurosurgery, Zhuzhou Central Hospital, Zhuzhou, Hunan 412007; \\ ${ }^{4}$ Department of Neurosurgery, Southwest Hospital, Third Military Medical University, Chongqing 400038, P.R. China; \\ ${ }^{5}$ Department of Physiology and Pharmacology, Loma Linda University, Loma Linda, CA 92354, USA
}

Received December 17, 2015; Accepted February 24, 2016

DOI: $10.3892 / e t m .2016 .3640$

\begin{abstract}
Hydrocephalus has been demonstrated to be an independent risk factor for poor outcomes in patients with subarachnoid hemorrhage (SAH). Blockage of cerebrospinal fluid (CSF) flow and drainage is widely considered to play a vital role in communicating hydrocephalus, possibly due to subarachnoid fibrosis. A previous study indicated that transforming growth factor- $\beta 1$ (TGF- $\beta 1$ ), a key fibrogenic factor, is significantly increased in the CSF following SAH, implying a pivotal role in the development of chronic hydrocephalus. To investigate whether LSKL peptide, a small molecular peptide and competitive antagonist for TGF- $\beta 1$, protects against subarachnoid fibrosis and hydrocephalus after $\mathrm{SAH}$, a two-hemorrhage injection model of SAH was created in Sprague-Dawley rats. LSKL ( $1 \mathrm{mg} / \mathrm{kg})$ was administered intraperitoneally immediately following the first intravenous injection of blood in the SAH model, with repeated injections of LSKL every $12 \mathrm{~h}$ until sacrifice. Thrombospondin-1 (TSP1), TGF- $\beta 1$, p-Smad2/3, collagen I and pro-collagen I c-terminal propeptide levels were assessed via western blotting and ELISA. Lateral ventricular index, Masson staining and Morris water maze tests were employed to evaluate subarachnoid fibrosis, hydrocephalus and long-term neurological function following SAH. It was found that the LKSL peptide readily crossed the blood brain barrier, was protective against subarachnoid fibrosis, attenuated ventriculomegaly and effectively suppressed hydrocephalus. In addition, the results indicated that the protective effects of the LSKL
\end{abstract}

Correspondence to: Dr Fei Liu, Department of Neurosurgery, The Third Xiangya Hospital, Central South University, 138 Tongzipo Road, Yuelu, Changsha, Hunan 410013, P.R. China E-mail: doctorlf@126.com

Key words: subarachnoid hemorrhage, LSKL peptide, subarachnoid fibrosis, hydrocephalus, transforming growth factor- $\beta 1$ peptide were achieved via the inhibition of TGF- $\beta 1$ activity and subsequent Smad2/3 signaling. Importantly, the LSKL peptide may improve long-term neurocognitive deficits after SAH. In conclusion, the LSKL peptide suppresses subarachnoid fibrosis via inhibition of TSP1-mediated TGF- $\beta 1$ activity, prevents the development of chronic hydrocephalus and improves long-term neurocognitive defects following SAH.

\section{Introduction}

Subarachnoid hemorrhage (SAH) is a fatal subtype of hemorrhagic stroke associated with significant morbidity and a high mortality rate of up to $50 \%$ (1). Hydrocephalus is an independent risk factor for poor outcomes in patients with SAH (2). Blockage of cerebrospinal fluid (CSF) flow and drainage is widely considered to play a vital role in communicating hydrocephalus (3), possibly by inducing subarachnoid fibrosis $(4,5)$. Therefore, to improve long-term neurological outcomes of patients with $\mathrm{SAH}$, it is important to develop new therapies for subarachnoid fibrosis and chronic hydrocephalus.

Antifibrinolytic therapy, commonly used for reducing the rate of further hemorrhage in patients with $\mathrm{SAH}$, has also been reported to be associated with hydrocephalus and delayed brain injury after SAH (6). A previous study by the authors of the present study indicated that transforming growth factor- $\beta 1$ (TGF- $\beta 1$ ), a key fibrogenic factor, is significantly elevated in the CSF after SAH (7), which implies a pivotal role in the development of chronic hydrocephalus $(8,9)$. TGF- $\beta$ is usually stored in the extracellular matrix by binding to latency-associated peptide (LAP). Thrombospondin-1 (TSP1) converts the latent TGF- $\beta /$ LAP complex to its active form by binding to LAP, releasing TGF- $\beta$, and making it available to bind to and activate the TGF- $\beta$ receptor (10).

Several previous studies have reported that a small peptide (molecular weight, 458.6 Da), the leucine-serine-lysine-leucine (LSKL) peptide, can inhibit the binding of TSP1 to LAP (11-13) and alleviate renal interstitial fibrosis (12) as well as hepatic fibrosis (13). It remains unclear, however, whether LSKL is protective against subarachnoid fibrosis and 
chronic hydrocephalus following SAH. Thus, the present study sought to investigate the potential protective role of LSKL in SAH-induced subarachnoid fibrosis, specifically whether it acts via inhibition of TGF- $\beta 1$ signaling, prevention of chronic hydrocephalus, and improvement in long-term cognitive deficits in a rat model of SAH.

\section{Materials and methods}

Animals. All experimental protocols were approved by the Ethics Committee of Central South University (Changsha, China), and all animal procedures were conducted in accordance with the eighth edition of the National Institutes of Health Guide for the Care and Use of Laboratory Animals (2011).

In total, 103 male Sprague-Dawley rats (age, 6 weeks; weight, 160-180 g; Experimental Center of Central South University) were used. These rats were divided into four groups: Sham group $(\mathrm{n}=34), \mathrm{SAH}+$ phosphate buffer solution (PBS) group $(\mathrm{n}=28), \mathrm{SAH}+\mathrm{N}^{15}$-labeled LSKL peptide $\left(\right.$ LSKL-N $\left.{ }^{15}\right)$ group $(n=12)$ and $\mathrm{SAH}+\mathrm{LSKL}$ group $(\mathrm{n}=29)$. All rats were kept in a quiet room at $23-25^{\circ} \mathrm{C}, 70 \%$ humidity and a $12 \mathrm{~h}$ light/dark cycle and were allowed free access to normal rat chow and filtered water.

SAH model. Rats were intubated transorally and then anesthetized and maintained under anesthesia with $3 \%$ isoflurane in 70:30 medical air:oxygen. The rats were then placed in a supine position on a heating pad to maintain the body temperature at $36.5 \pm 0.5^{\circ} \mathrm{C}$. The SAH model was executed according to the two-hemorrhage cisterna magna method as previously described (14). Briefly, a small $(1.0-1.5 \mathrm{~cm})$ longitudinal, midline suboccipital incision was made over the center of the foramen magnum, and the neck muscles were dissected until the dura was visible. Autologous unheparinized blood $(0.5 \mathrm{ml})$ drawn from the arteria cruralis was injected into the cisterna magna (defined as day 0 ) with a 25 -gauge butterfly needle. The rat was then placed on an inclined board at a $45^{\circ}$ angle with the head down in a neutral position for $30 \mathrm{~min}$. A second injection of blood was conducted $24 \mathrm{~h}$ later by the same procedure. Sham-operated animals were treated in a similar manner with the exception that $0.5 \mathrm{ml}$ PBS was injected instead of $0.5 \mathrm{ml}$ unheparinized blood.

The severity of SAH was assessed using an 18-point SAH grading scale as previously reported (15). The score was based on the amount of subarachnoid blood clot. Each of six segments of the basal cistern were scored on a scale from 0 to 3 (total, 0 -18 points). If the score is $<8$, then the animal was excluded from the study. Previous and present studies conducted in the current team's laboratory demonstrate minimal variability in the scoring of SAH (16-18).

LSKL peptide administration and detection. To detect the ability of LSKL to pass the blood brain barrier, isotope-labeled LSKL peptide (LSKL-N ${ }^{15}$; GL Biochem Ltd., Shanghai, China), $1 \mathrm{mg} / \mathrm{kg}$ (11), was injected intraperitoneally on day 3 after SAH $(\mathrm{n}=10)$. At $5 \mathrm{~min}$ after administration, $100 \mu \mathrm{l}$ clear and blood-free CSF was collected via cisterna magna puncture under microsurgery with a 27-gauge needle and immediately frozen on dry ice and maintained at $-80^{\circ} \mathrm{C}$ until analysis. At the same time, $1 \mathrm{ml}$ fresh venous blood was collected from the left femoral vein and centrifuged at $4^{\circ} \mathrm{C}$ and $12,000 \mathrm{x} \mathrm{g}$ for $10 \mathrm{~min}$. The supernatant was collected and maintained at $-80^{\circ} \mathrm{C}$ until analysis. The concentration of LSKL-N ${ }^{15}$ was detected by stable isotope ratio mass spectrometry (Finnigan MAT 253; Thermo Fisher Scientific, Inc., Waltham, MA, USA).

For functional assessment of the LSKL peptide, LSKL $(1 \mathrm{mg} / \mathrm{kg}$ ) was intraperitoneally administered immediately following the first blood injection of the SAH modeling procedure and repeated once every $12 \mathrm{~h}$ until sacrifice.

Morris water maze. Between days 17 and 20 following SAH, the Morris water maze test ( $n=13$ per group) was performed in a blinded setup to evaluate SAH-induced neurocognitive deficits, as previously described (19). Briefly, the test consisted of three trials, including a cued learning paradigm, spatial paradigm and probe paradigm. All trials lasted a maximum of $60 \mathrm{sec}$.

The cued learning trials were conducted on day 17 after SAH for non-associative factors that could affect scoring on the Morris water maze, such as motivation, swimming ability and vision. During the cued learning trials, there was a visible platform above the water surface. Rats were placed in the tank and required simply to swim to the platform to end the trial, and were allowed to remain on the platform for $10 \mathrm{sec}$ after finding or being guided to it.

On the following 3 consecutive days, the spatial and probe trials were conducted to measure the ability of the rat to learn and remember the location of a hidden platform in the tank. Unlike the cued learning trial, the platform in these trials was submerged under the water. Once a rat was released in the tank, it was allowed to swim and search for the platform. The total distance to find the platform was measured to reflect spatial learning ability. At $1 \mathrm{~h}$ after the spatial trial, the platform was removed completely and the rats were allowed to swim again in search of the now-absent platform. The percentage of time spent in the probe quadrant (the previous location of the platform) was measured to reflect spatial memory ability.

Lateral ventricle index calculation. On day 21 after SAH, rats $(\mathrm{n}=10$, shared with Morris water maze test) were transcardially perfused with ice-cold PBS ( $\mathrm{pH} 7.4$ ), followed by perfusion with $4 \%$ paraformaldehyde. The brains were then removed and fixed in $4 \%$ paraformaldehyde at $4^{\circ} \mathrm{C}$ for 3 days. Following dehydration with $30 \%$ sucrose in PBS ( $\mathrm{pH} 7.4)$, the brains were cut into $10 \mu \mathrm{m}$ sections on a vibratome.

The size of the lateral ventricle was determined using the lateral ventricle index, which was calculated on the basis of measurements of the brain slices using ImageJ software (version 1.48; National Institutes of Health, Bethesda, MD, USA). Specifically, the lateral ventricle index was calculated as the lateral ventricle volume divided by the total area of the brain slice at the level of the preoptic chiasm. Hydrocephalus was defined as a lateral ventricle index $>3$ standard deviations above the mean in sham animals (20).

Masson staining. The brain samples ( $\mathrm{n}=3$, shared with Morris water maze test) were prepared in a manner similar to that of the animals used for the lateral ventricle index calculation, with the exception that brain tissue from the dura mater was 
not removed in order to prevent possible traction injury of the leptomeninges. Masson staining was performed according to the manufacturer's protocol (Leagene Biotech Co., Ltd., Beijing, China). Briefly, the brain slices were stained with Masson composite staining solution for $5 \mathrm{~min}$, washed briefly with $0.2 \%$ acetic acid solution, for $5 \mathrm{~min}$ with $5 \%$ phosphotungstic acid, and twice briefly with $0.2 \%$ acetic acid. Then, the slices were stained with $1 \%$ aniline blue for $5 \mathrm{~min}$, followed by washing twice briefly with $0.2 \%$ acetic acid. The slices were then dehydrated in absolute alcohol, rendered transparent by placing in xylene, and finally mounted with neutral gum. Collagen fibers appeared bluish green in color when observed under a light microscope.

Enzyme-linked immunosorbent assay (ELISA). On days 3, 4 and 5 after SAH, $100 \mu \mathrm{l} \mathrm{CSF}$ was collected $(\mathrm{n}=10) 5 \mathrm{~min}$ after LSKL administration. These CSF samples were mixed and divided into three equal shares for the detection of TSP1, activated TGF- $\beta 1$ and total TGF- $\beta 1$ using the respective ELISA kit (Wuhan Boster Biological Technology, Ltd., Wuhan, China). For total TGF- $\beta 1$ detection, $1 \mathrm{~mol} / 1 \mathrm{HCl}$ was added to the CSF at the ratio of $3: 1$ for $10 \mathrm{~min}$. The sample was then analyzed using the ELISA kit used to detect activated TGF- $\beta 1$.

On day 21 after SAH, $100 \mu \mathrm{l}$ CSF was collected from each of the rats used for the Morris water maze test $(n=10)$. The content of pro-collagen I c-terminal propeptide (PICP) was then detected using a PICP ELISA kit (Wuhan Boster Biological Technology, Ltd.).

Western blotting. Western blot analysis for superficial brain tissues was performed as previously described $(21,22)$. Brain tissues were isolated on day 5 after SAH $(n=10)$ and homogenized in a lysis buffer containing $150 \mathrm{mmol} / \mathrm{l} \mathrm{NaCl}, 50 \mathrm{mmol} / \mathrm{l}$ Tris- $\mathrm{HCl}, 10 \mathrm{mmol} / 1$ ethylenediamine tetra-acetic acid, $0.1 \%$ Tween-20, $1 \%$ Triton X-100, 0.1\% $\beta$-mercaptoethanol, $0.1 \mathrm{mmol} / 1$ phenylmethylsulfonyl fluoride, $5 \mu \mathrm{g} / \mathrm{ml}$ leupeptin and $5 \mu \mathrm{g} / \mathrm{ml}$ aprotinin, $\mathrm{pH}$ 7.4. Homogenates were centrifuged at $4^{\circ} \mathrm{C}$ for $10 \mathrm{~min}$ at $10,000 \mathrm{x} \mathrm{g}$, and supernatants were collected. Protein concentrations were determined using a protein assay kit (Bio-Rad Laboratories, Inc., Hercules, CA). Following this, the sample $(50 \mu \mathrm{g})$ was loaded onto $10 \%$ polyacrylamide gel with $0.1 \%$ sodium dodecyl sulfate and separated by electrophoresis at $100 \mathrm{~V}$ for $120 \mathrm{~min}$. Proteins were then transferred onto nitrocellulose membranes and probed with primary antibodies against collagen I (1:2,000; sc-59772; Santa Cruz Biotechnology, Inc., Dallas, TX, USA), Smad2/3 and phospho (p)-Smad2/3 (1:1,000; sc-376928 and sc-101801, respectively; Santa Cruz Biotechnology, Inc.). After washing, membranes were incubated with secondary horseradish peroxidase (HRP)-conjugated goat anti-rabbit antibody and HRP-conjugated goat anti-mouse antibody (1:4,000; A0208 and A0216; Beyotime Institute of Biotechnology, Shanghai, China). Proteins were visualized with enhanced chemiluminescence reagents (ECL Plus; GE Healthcare Life Sciences, Chalfont, UK), and blots were exposed to Hyperfilm. The results were analyzed with Kodak ID image analysis software (Kodak, Rochester, NY, USA). The relative intensity of the bands of interest was calculated by correcting for $\beta$-actin (1:4,000; sc-47778; Santa Cruz Biotechnology, Inc.) from the same sample. Fold changes were then calculated against control intensities from sham-treated animals.
Statistical analysis. Statistical analysis was performed using GraphPad Prism 5 (GraphPad Software, Inc., La Jolla, CA, USA) and SPSS version 16.0 software (SPSS, Inc., Chicago, IL, USA). Data were expressed as the mean \pm standard error of the mean and analyzed by one-way analysis of variance followed by Student-Newman-Keuls test. Mortality data were analyzed by Fisher's exact test. $\mathrm{P}<0.05$ was considered to indicate a statistically significant difference.

\section{Results}

Mortality. Of the 103 rats in the present study, 14 rats died and were excluded from further experiments. One rat in the sham group died during surgery. Five rats in the SAH+PBS group, 2 rats in the SAH+LSKL-N ${ }^{15}$ group and 6 rats in the SAH+LSKL group died within $72 \mathrm{~h}$ after SAH model initiation, perhaps due to severe brain injury. The total mortality rates were $17.8,16.7$ and $20.7 \%$ in the SAH+PBS, SAH+LSKL-N ${ }^{15}$ and SAH+LSKL groups, respectively, with no significant differences between the groups $(\mathrm{P}>0.05)$.

LSKL peptide can easily traverse the blood brain barrier. At 5 min after the intraperitoneal injection of $\mathrm{N}^{15}$-labelled LSKL into the rats, the concentration of LSKL-N ${ }^{15}$ was lower in the CSF than in the blood in sham rats $(\mathrm{P}<0.05)$; however, there was no significant difference in LSKL-N ${ }^{15}$ concentration between the CSF and blood in the SAH group (Fig. 1A). The ratio of LSKL-N ${ }^{15}$ between the CSF and blood significantly increased after $\mathrm{SAH}$ in comparison with that in the sham group $(\mathrm{P}<0.05)$ by a mean value of $84.99 \%$ (Fig. 1B).

LSKL peptide alleviates hydrocephalus and long-term cognitive deficits after SAH. PBS-treated rats following SAH exhibited a significantly larger lateral ventricular index compared with the sham rats $(\mathrm{P}<0.05)$. Further analysis revealed that LSKL treatment reduced the lateral ventricular index, an indicator of ventriculomegaly, compared with that in the SAP+PBS group $(\mathrm{P}<0.05$; Fig. 1C).

The Morris water maze results indicate that the two-hemorrhage injection rat model of SAH induced significant increases in latency to reach the platform on days 18,19 and 20 after SAH $(\mathrm{P}<0.05$; Fig. 1D), decreased the total swim distance $(\mathrm{P}<0.05$; Fig. 1E), and increased the number of times that the rat entered the platform quadrant $(\mathrm{P}<0.05$; Fig. $1 \mathrm{~F})$. However, treatment with the LSKL peptide attenuated these unfavorable effects when compared with the SAH+PBS group ( $\mathrm{P}<0.05$; Fig. 1D-F).

LSKL peptide inhibits subarachnoid fibrosis after SAH. On day 21 after $\mathrm{SAH}$, Masson staining revealed greater quantities of collagen fibers in the brains of rats in the SAH+PBS group as compared with the SAH+LSKL group (Fig. 2A). In addition, as compared with the sham group, PICP levels in the CSF were significantly elevated on day 21 in the SAH+PBS and SAH+LSKL treatment groups $(\mathrm{P}<0.05$; Fig. 2B). Notably, LSKL treatment effectively reduced the concentration of PICP compared with that in the SAH+PBS group ( $\mathrm{P}<0.05$; Fig. $2 \mathrm{~B})$. Furthermore, on day 5 after $\mathrm{SAH}$, the expression level of collagen I in the SAH+PBS group was significantly increased in comparison with that in the sham group $(\mathrm{P}<0.05)$ but was reduced by LSKL treatment $(\mathrm{P}<0.05$; Fig. $2 \mathrm{C})$. 

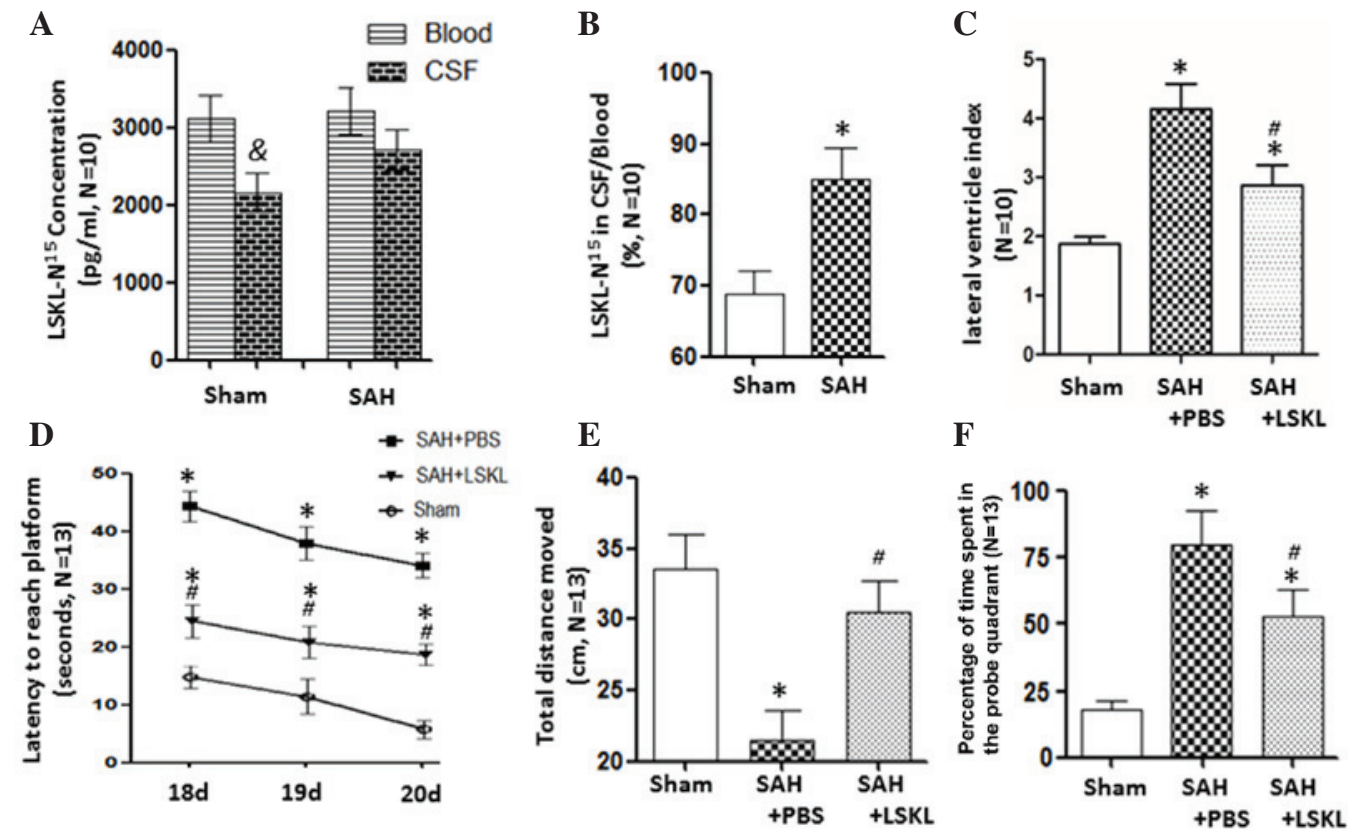

Figure 1. LSKL peptide alleviated hydrocephalus and improved long-term cognitive function following SAH. (A) Quantitative analysis of $\mathrm{N}^{15}$-labelled LSKL peptide in the blood and CSF on day 3 after SAH. (B) Ratio of $\mathrm{N}^{15}$-labelled LSKL peptide between the CSF and blood. (C) Quantitative analysis of the lateral ventricular index on day 21 after SAH. Quantitative analysis of (D) the latency to reach the platform, (E) the total swim distance to the platform and (F) the percentage of time rats spent in the probe quadrant in the Morris water maze test from day 18 to day 20 after SAH. Data are expressed as the mean \pm standard error of the mean ( $\mathrm{n}=13$ for Morris water maze test, $\mathrm{n}=10$ for other results). $\mathrm{P}<0.05$ vs. the sham group; ${ }^{\#} \mathrm{P}<0.05$ vs. the $\mathrm{SAH}+\mathrm{PBS}$ group; ${ }^{\text {\& }} \mathrm{P}<0.05$ vs. the value in blood. SAH, subarachnoid hemorrhage; CSF, cerebrospinal fluid; LSKL, leucine-serine-lysine-leucine; PBS, phosphate buffer solution.

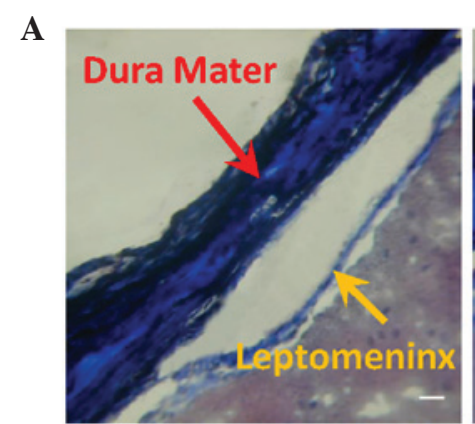

Sham

B

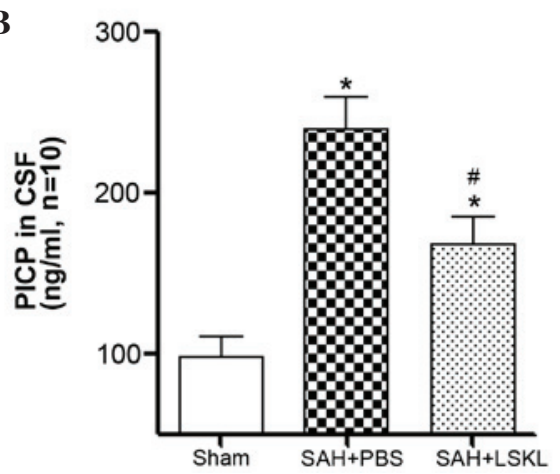

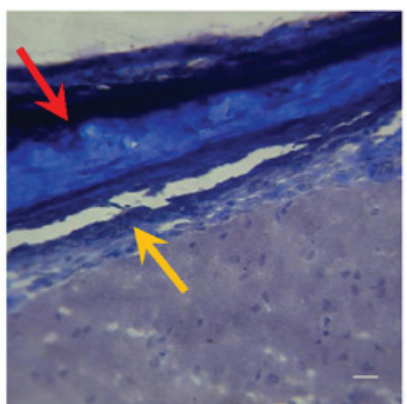

SAH+PBS

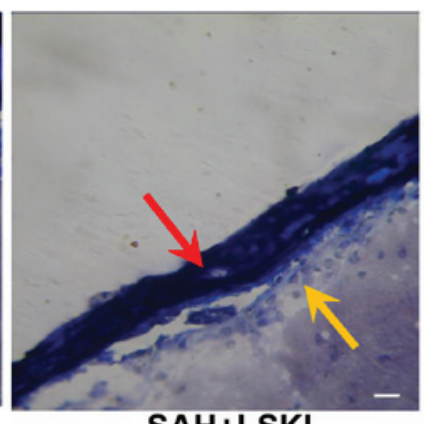

SAH+LSKL

C
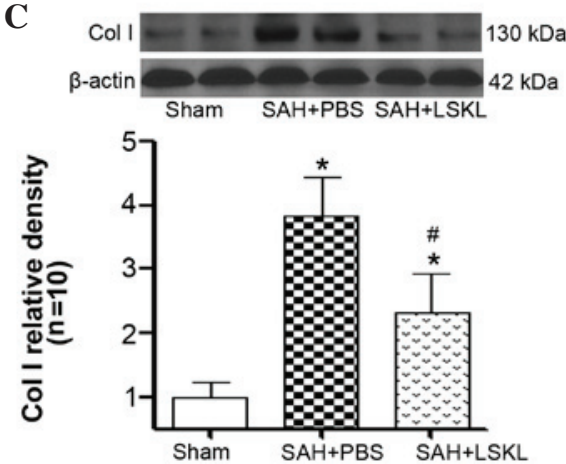

Figure 2. LSKL peptide suppressed subarachnoid fibrosis following SAH. (A) Representative Masson staining slices of rat brain on day 21 after SAH (n=3; scale bar, $100 \mu \mathrm{m}$ ). (B) Quantitative analyses of PICP in CSF at day 21 after SAH. (C) Representative western blot bands and quantitative analyses of collagen I on day 5 after SAH. Relative densities of each protein have been normalized against the sham group. Data are expressed as the mean \pm standard error of the mean $(\mathrm{n}=10)$. " $\mathrm{P}<0.05$ vs. the sham group; ${ }^{\text {P }}<0.05$ vs. the $\mathrm{SAH}+\mathrm{PBS}$ group. $\mathrm{SAH}$, subarachnoid hemorrhage; CSF, cerebrospinal fluid; PICP, pro-collagen I c-terminal propeptide; Col I, collagen I; LSKL, leucine-serine-lysine-leucine; PBS, phosphate buffer solution.

LSKL peptide inhibits TGF- $\beta 1$ signaling activity after SAH. The concentration of TSP1 in the CSF of rats in the SAH+saline group was significantly increased compared with that in the sham group $(\mathrm{P}<0.05)$, and LSKL treatment only slightly reduced the concentration of TSP1 following SAH $(\mathrm{P}>0.05$; Fig. 3A). However, LSKL treatment significantly decreased 
A

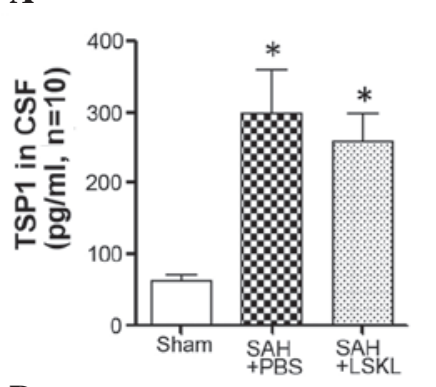

D

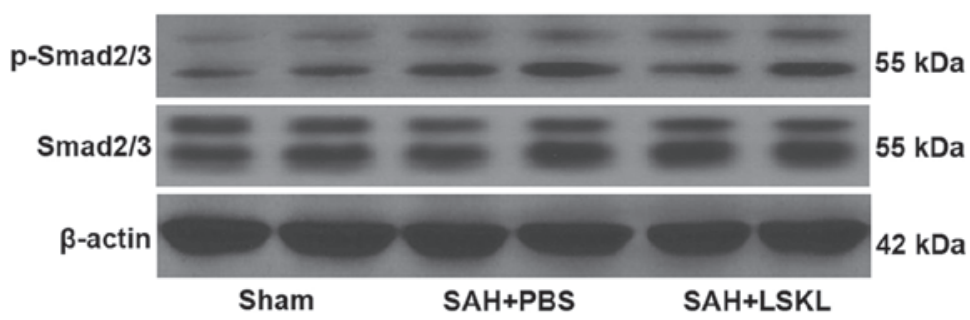

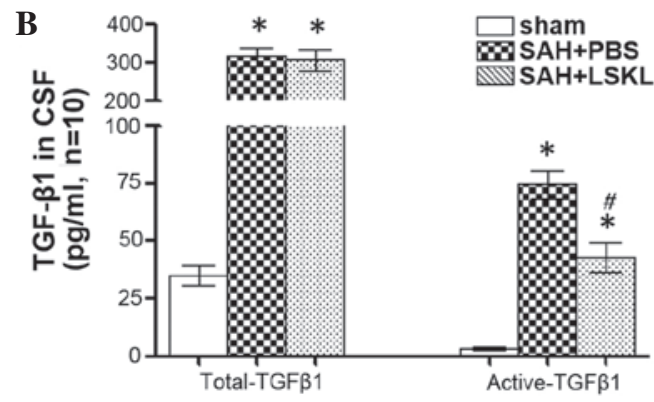

$\mathrm{C}$

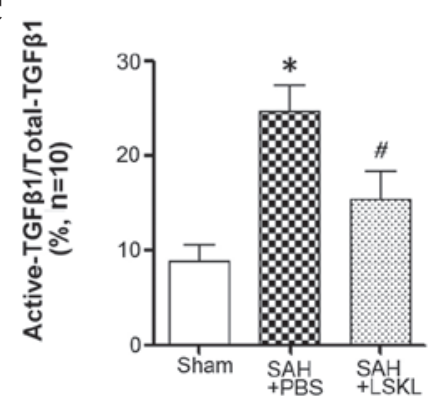

E

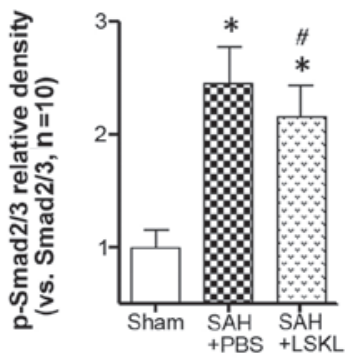

Figure 3. LSKL peptide inhibited TSP1-mediated TGF- $\beta 1$ signaling activity following SAH. Quantitative analyses of (A) TSP1 and (B) total TGF- $\beta 1$ and active TGF- $\beta 1$ in the CSF on days 3-5 after SAH. (C) Ratio of active TGF- $\beta 1$ to total TGF- $\beta 1$ in the CSF on days 3-5 after SAH. (D) Representative western blot bands of $\mathrm{p}-\mathrm{Smad} 2 / 3$ and (E) quantitative analyses of p-Smad2/3 expression on day 5 after SAH. Relative densities of each protein have been normalized against the sham group. Data are expressed as the mean \pm standard error of the mean $(n=10)$. ${ }^{*} \mathrm{P}<0.05$ vs. the sham group; ${ }^{*} \mathrm{P}<0.05$ vs. the $\mathrm{SAH}+\mathrm{PBS}$ group. SAH, subarachnoid hemorrhage; CSF, cerebrospinal fluid; TSP1, thrombospondin-1; LSKL, leucine-serine-lysine-leucine; PBS, phosphate buffer solution.

the activation of TGF- $\beta 1$ in the CSF in comparison with that in the SAH+PBS group ( $\mathrm{P}<0.05$; Fig. 3B and $\mathrm{C})$. Moreover, the expression of $\mathrm{p}-\mathrm{Smad} 2 / 3$ in the rat brains was significantly increased at day 5 after $\mathrm{SAH}(\mathrm{P}<0.05)$, and treatment with LSKL significantly reduced the elevated level of $\mathrm{p}-\mathrm{Smad} 2 / 3$ following SAH in comparison with that in the SAH+PBS group $(\mathrm{P}<0.05$; Fig. 3D and $\mathrm{E})$.

\section{Discussion}

In the present study, it was demonstrated that LKSL peptide easily crosses the blood brain barrier and protects against subarachnoid fibrosis, attenuates ventriculomegaly, and effectively suppresses the development of chronic hydrocephalus in a rat model of SAH. In addition, it was revealed that the protective effects of LSKL peptide are achieved primarily via inhibition of the activating process of TGF- $\beta 1$ and downstream Smad2/3 signaling without affecting the expression of TSP1, which is critically implicated in the pathogenesis of subarachnoid fibrosis and chronic hydrocephalus after SAH (11-13). Notably, it was also demonstrated that the administration of LSKL peptide ameliorates long-term neurocognitive deficits following SAH.

Early brain injury is currently considered the most promising target in the treatment of SAH (23); however, delayed neurological deterioration remains one of the major causes of severe morbidity (24). Although it presents less frequently than other complications of $\mathrm{SAH}$, chronic communicating hydrocephalus reportedly contributes to poor long-term neurological outcomes, particularly severe cognitive deficits (25). Two-hemorrhage injection models of SAH were employed in the present study, which produce a similar incidence of hydrocephalus to other SAH models $(26,27)$. Evidence from previous studies indicates that fibrosis of the leptomeninges and arachnoid granulations due to blood product deposition may contribute to the development of post-hemorrhagic communicating hydrocephalus via blockage of the flow of CSF, suppression of CSF absorption, and reduction of CSF drainage $(28,29)$.

TGF- $\beta$ is a potent fibrogenic factor in the pathogenesis of fibrosis, with an important role in various cellular processes such as cell proliferation, differentiation, apoptosis, migration, and stimulation of extracellular matrix synthesis. Members of the TGF- $\beta$ family can bind to TGF- $\beta$ type- 1 and type- 2 receptors on the cell surface, inducing phosphorylation of Smad2/3, initiating multiple intracellular signaling and exerting pro-fibrotic effects (30). Marked upregulation of TGF- $\beta 1$ has been observed in the CSF of patients following SAH, particularly in those with hydrocephalus $(9,31)$. However, only trace amounts of TGF- $\beta 1$ are found in the CSF of healthy individuals, although platelets are known to store excess TGF- $\beta 1$, which can be released by platelet degranulation after aneurysm rupture to initiate the local synthesis of extracellular matrix (31). In the present study, an increase of TGF- $\beta 1$ in the CSF of SAH model rats was observed. This is consistent with the previous study in which the active form of TGF- $\beta 1$ was shown to be vital in activating TGF- $\beta 1 / \mathrm{Smad} 2 / 3$ and the downstream biological effects.

One of the key regulatory factors of TGF- $\beta$ activity is its conversion from the latent precursor to the biologically active form. The activation of TGF- $\beta$ is dependent on the interaction of a specific amino acid sequence $\left(\mathrm{K}^{412} \mathrm{RFK}^{415}\right)$ in TSP1, with the latent TGF-LAP complex (10). Formation of the TSP1-LAP complex requires the activation sequence Lys-Arg-Phe-Lys (KRFK) and a sequence (LSKL) near the amino terminus of LAP, which is also conserved in TGF- $\beta$. Endogenous LSKL peptides are able to competitively inhibit the activation by TSP or other KRFK sequence-containing peptides (32). In addition, the interaction of LAP with TSP1 may prevent the reformation of an inactive TGF-LAP complex because TSP-bound LAP is 
not able to activate latent TGF- $\beta$ (32). In the present study, it was demonstrated that exogenous LSKL is capable of significantly reducing the activation of latent TGF- $\beta$ following SAH without affecting the expression of TSP1 in the CSF. The experimental data further demonstrate the potential of LSKL peptide to inhibit fibrosis, which is consistent with previous studies of fibrosis in other tissues $(11-13,33)$.

Exogenous LSKL peptide is a small peptide that consists of only four amino acids. Its simple structure and small molecular weight allow the unsaturated, exogenous LSKL peptide to easily cross the cell membrane and directly enter the cell without expending energy. Therefore, the absorption, transformation and utilization of LSKL peptide are efficient, complete and thorough, characteristics that may be favorable for clinical application to reduce subarachnoid fibrosis after SAH. In the present study, it was demonstrated that LSKL can efficiently cross the blood brain barrier, alleviate the symptoms of hydrocephalus, and ameliorate long-term neurocognitive deficits after SAH.

In summary, the present study indicates that LSKL peptide suppresses subarachnoid fibrosis via inhibition of TSP1-mediated TGF- $\beta 1$ activity, prevents the development of chronic hydrocephalus, and attenuates long-term neurocognitive deficits following SAH. Although direct translational trials of the present study may prove impractical, given the relatively high incidence and sustained debilitating properties of chronic hydrocephalus, LSKL peptide represents a potentially feasible and promising therapeutic tool. Further investigation of the protective effects of LSKL peptide in post-hemorrhagic chronic hydrocephalus is warranted.

\section{Acknowledgements}

This study was supported by the National Natural Science Foundation of China (grant no. 81571150 to Fei Liu, grant no. 81501002 to Yujie Chen and grant no. 81220108009 to Hua Feng) and the Science and Technology Planning Project of Hunan (grant no. 2012FJ3125 to Fei Liu).

\section{References}

1. Fujii M, Yan J, Rolland WB, Soejima Y, Caner B and Zhang JH: Early brain injury, an evolving frontier in subarachnoid hemorrhage research. Transl Stroke Res 4: 432-446, 2013.

2. Giraldo EA, Mandrekar JN, Rubin MN, Dupont SA, Zhang Y, Lanzino G, Wijdicks EF and Rabinstein AA: Timing of clinical grade assessment and poor outcome in patients with aneurysmal subarachnoid hemorrhage. J Neurosurg 117: 15-19, 2012.

3. Shah AH and Komotar RJ: Pathophysiology of acute hydrocephalus after subarachnoid hemorrhage. World Neurosurg 80 304-306, 2013.

4. Botfield H, Gonzalez AM, Abdullah O, Skjolding AD, Berry M, McAllister JP II and Logan A: Decorin prevents the development of juvenile communicating hydrocephalus. Brain 136: 2842-2858, 2013.

5. Ishii M, Suzuki S, Iwabuchi T and Julow J: Effect of antifibrinolytic therapy on subarachnoid fibrosis in dogs after experimental subarachnoid haemorrhage. Acta Neurochir (Wien) 54: 17-24, 1980.

6. Graff-Radford NR, Torner J, Adams HP Jr and Kassell NF: Factors associated with hydrocephalus after subarachnoid hemorrhage. A report of the Cooperative Aneurysm Study. Arch Neurol 46: 744-752, 1989.

7. Liu F, Yuan W, Liao D, Zhang T and Wang Z: Association of chronic hydrocephalus after aneurysmal subarachnoid hemorrhage with transforming growth factor- $\beta 1$ levels and other risk factors Nan Fang Yi Ke Da Xue Xue Bao 33: 382-385, 2013 (In Chinese).
8. Li T, Zhang P, Yuan B, Zhao D, Chen $\mathrm{Y}$ and Zhang $\mathrm{X}$ : Thrombin-induced TGF- $\beta 1$ pathway: A cause of communicating hydrocephalus post subarachnoid hemorrhage. Int J Mol Med 31: 660-666, 2013.

9. Douglas MR, Daniel M, Lagord C, Akinwunmi J, Jackowski A, Cooper C, Berry M and Logan A: High CSF transforming growth factor beta levels after subarachnoid haemorrhage: Association with chronic communicating hydrocephalus. J Neurol Neurosurg Psychiatry 80: 545-550, 2009.

10. Ribeiro SM, Poczatek M, Schultz-Cherry S, Villain M and Murphy-Ullrich JE: The activation sequence of thrombospondin-1 interacts with the latency-associated peptide to regulate activation of latent transforming growth factor-beta. J Biol Chem 274: 13586-13593, 1999.

11. Kuroki H, Hayashi H, Nakagawa S, Sakamoto K, Higashi T, Nitta H, Hashimoto D, Chikamoto A, Beppu T and Baba H: Effect of LSKL peptide on thrombospondin 1-mediated transforming growth factor $\beta$ signal activation and liver regeneration after hepatectomy in an experimental model. Br J Surg 102: 813-825, 2015.

12. Xie XS, Li FY, Liu HC, Deng Y, Li Z and Fan JM: LSKL, a peptide antagonist of thrombospondin-1, attenuates renal interstitial fibrosis in rats with unilateral ureteral obstruction. Arch Pharm Res 33: 275-284, 2010.

13. Kondou H, Mushiake S, Etani Y, Miyoshi Y, Michigami T and Ozono K: A blocking peptide for transforming growth factor-betal activation prevents hepatic fibrosis in vivo. J Hepatol 39: 742-748, 2003.

14. Zhang J, Xu X,Zhou D, Li H, You W, Wang Z and Chen G: Possible role of raf-1 kinase in the development of cerebral vasospasm and early brain injury after experimental subarachnoid hemorrhage in rats. Mol Neurobiol 52: 1527-1539, 2015.

15. Sugawara T, Ayer R, Jadhav V and Zhang JH: A new grading system evaluating bleeding scale in filament perforation subarachnoid hemorrhage rat model. J Neurosci Methods 167: 327-334, 2008.

16. Yan H, Chen Y, Li L, Jiang J, Wu G, Zuo Y, Zhang JH, Feng H, Yan X and Liu F: Decorin alleviated chronic hydrocephalus via inhibiting TGF- $\beta 1 / \mathrm{Smad} / \mathrm{CTGF}$ pathway after subarachnoid hemorrhage in rats. Brain Res 1630: 241-253, 2016.

17. Liu F, Chen Y, Hu Q, Li B, Tang J, He Y, Guo Z, Feng H, Tang $\mathrm{J}$ and $\mathrm{Zhang} \mathrm{JH}$ : MFGE8/Integrin $\beta 3$ pathway alleviates apoptosis and inflammation in early brain injury after subarachnoid hemorrhage in rats. Exp Neurol 272: 120-127, 2015.

18. Liu F, Hu Q, Li B, Manaenko A, Chen Y, Tang J, Guo Z, Tang J and Zhang JH: Recombinant milk fat globule-EGF factor- 8 reduces oxidative stress via integrin $\beta 3 /$ nuclear factor erythroid 2-related factor $2 /$ heme oxygenase pathway in subarachnoid hemorrhage rats. Stroke 45: 3691-3697, 2014.

19. Hu Q, Liang X, Chen D, Chen Y, Doycheva D, Tang J, Tang $J$ and Zhang JH: Delayed hyperbaric oxygen therapy promotes neurogenesis through reactive oxygen species/hypoxia-inducible factor- $1 \alpha / \beta$-catenin pathway in middle cerebral artery occlusion rats. Stroke 45: 1807-1814, 2014.

20. Okubo S, Strahle J, Keep RF, Hua Y and Xi G: Subarachnoid hemorrhage-induced hydrocephalus in rats. Stroke 44: 547-550, 2013

21. Chen Y, Luo C, Zhao M, Li Q, Hu R, Zhang JH, Liu Z and Feng H: Administration of a PTEN inhibitor BPV(pic) attenuates early brain injury via modulating AMPA receptor subunits after subarachnoid hemorrhage in rats. Neurosci Lett 588: 131-136, 2015.

22. Chen Y, Zhang Y, Tang J, Liu F, Hu Q, Luo C, Tang J, Feng H and Zhang JH: Norrin protected blood-brain barrier via frizzled-4/ $\beta$-catenin pathway after subarachnoid hemorrhage in rats. Stroke 46: 529-536, 2015.

23. Sehba FA, Hou J, Pluta RM and Zhang JH: The importance of early brain injury after subarachnoid hemorrhage. Prog Neurobiol 97: 14-37, 2012.

24. Macdonald RL: Delayed neurological deterioration after subarachnoid haemorrhage. Nat Rev Neurol 10: 44-58, 2014.

25. Chen S, Feng H, Sherchan P, Klebe D, Zhao G, Sun X, Zhang J, Tang J and Zhang JH: Controversies and evolving new mechanisms in subarachnoid hemorrhage. Prog Neurobiol 115: 64-91, 2014.

26. Lackner P, Vahmjanin A, Hu Q, Krafft PR, Rolland W and Zhang JH: Chronic hydrocephalus after experimental subarachnoid hemorrhage. PLoS One 8: e69571, 2013. 
27. Chu SH, Feng DF, Ma YB, Zhang H, Zhu ZA, Li ZQ and Zhang ZH: Expression of HGF and VEGF in the cerebral tissue of adult rats with chronic hydrocephalus after subarachnoid hemorrhage. Mol Med Rep 4: 785-791, 2011.

28. Strahle J, Garton HJ, Maher CO, Muraszko KM, Keep RF and Xi G: Mechanisms of hydrocephalus after neonatal and adult intraventricular hemorrhage. Transl Stroke Res 3 (Suppl 1): S25-S38, 2012.

29. Orešković D and Klarica M: Development of hydrocephalus and classical hypothesis of cerebrospinal fluid hydrodynamics: Facts and illusions. Prog Neurobiol 94: 238-258, 2011.

30. Lee HS: Paracrine role for TGF- $\beta$-induced CTGF and VEGF in mesangial matrix expansion in progressive glomerular disease. Histol Histopathol 27: 1131-1141, 2012.
31. Flood C, Akinwunmi J, Lagord C, Daniel M, Berry M, Jackowski A and Logan A: Transforming growth factor-betal in the cerebrospinal fluid of patients with subarachnoid hemorrhage: Titers derived from exogenous and endogenous sources. J Cereb Blood Flow Metab 21: 157-162, 2001.

32. Young GD and Murphy-Ullrich JE: Molecular interactions that confer latency to transforming growth factor-beta. J Biol Chem 279: 38032-38039, 2004

33. Krishna SM, Seto SW, Jose RJ, Biros E, Moran CS, Wang Y, Clancy $\mathrm{P}$ and Golledge J: A peptide antagonist of thrombospondin-1 promotes abdominal aortic aneurysm progression in the angiotensin II-infused apolipoprotein-E-deficient mouse. Arterioscler Thromb Vasc Biol 35: 389-398, 2015. 